\title{
E2F1-inducible microRNA 449a/b suppresses cell proliferation and promotes apoptosis
}

\author{
M Lizé ${ }^{1}$, S Pilarski ${ }^{2}$ and M Dobbelstein ${ }^{*, 1}$
}

E2F1 is a positive regulator of cell cycle progression and also a potent inducer of apoptosis, especially when activated by DNA damage. We identified E2F1-inducible microRNAs (miRNAs) by microarray hybridization and found that the levels of miRNAs $449 \mathrm{a}$ and $449 \mathrm{~b}$, as well as their host gene CDC20B, are strongly upregulated by E2F1. High miR-449 levels were found in testes, lung, and trachea, but not in testicular and other cancer cells. MiR-449a/b structurally resemble the p53-inducible miRNA 34 family. In agreement with a putative tumor-suppressive role, miR-449a as well as miR-34a reduced proliferation and strongly promoted apoptosis by at least partially p53-independent mechanisms. Both miRNAs reduced the levels of CDK6, implying miR449 in a negative feedback mechanism for E2F1. Moreover, miR-449a and miR-34a diminished the deacetylase Sirt1 and augmented p53 acetylation. We propose that both miRNAs provide a twofold safety mechanism to avoid excessive E2F1-induced proliferation by cell cycle arrest and by apoptosis. While responding to different transactivators, miRNAs 449 and 34 each repress E2F1, but promote p53 activity, allowing efficient cross-talk between two major DNA damage-responsive gene regulators.

Cell Death and Differentiation (2010) 17, 452-458; doi:10.1038/cdd.2009.188; published online 4 December 2009

The E2F family of transcription factors is essential for cell cycle progression, and repressing E2F activity is the key mechanism by which the retinoblastoma $(\mathrm{Rb})$ family of pocket proteins exerts its tumor-suppressive function. Cyclin-dependent kinases (cdks) phosphorylate and thereby inactivate pocket proteins, allowing cell proliferation, but this in turn is controlled by cdk inhibitors. Thus, E2Fs integrate cell cycle regulatory signals into a program of gene expression. In addition to cell cycle regulation, E2F proteins are also capable of inducing programmed cell death. In particular, E2F1 induces a number of target genes that mediate apoptosis. This happens mostly in the context of DNA damage, resulting in E2F1 phosphorylation and activation. ${ }^{1,2}$

This property of E2F1 is shared by the tumor suppressor p53. On DNA damage, p53 is phosphorylated and stabilized, along with enhanced activation of p53 target genes. However, the analogy in the functions of E2F1 and p53 goes beyond the mode of activation. E2F1 strongly upregulates the levels of TAp73, ${ }^{3,4}$ a p53-homologue that shares many activities of $\mathrm{p} 53$, and induces $\mathrm{p} 14$ arf, ${ }^{5}$ a positive regulator of $\mathrm{p} 53$. Independently of p53, E2F1 also activates the expression of Noxa and other BH3-only gene products, ${ }^{6}$ and these proapoptotic target genes are shared with p53. Thus, both E2F1 and p53 are strong inducers of apoptosis, at least in part by transactivating an overlapping but not identical set of target genes. ${ }^{7}$

On the other hand, p53 activity results in negative regulation of E2F1. Most notably, p53 induces the expression of the cdk inhibitor p21, thus leading to the accumulation of hypophosphorylated, active $\mathrm{Rb}$ pocket proteins and thus attenuated E2F1 activity. Hence, although E2F1 activity enhances p53 activity, p53 synergizes only with the proapoptotic activity of E2F1, while antagonizing E2F1-induced cell cycle progression. ${ }^{7}$

An important set of p53 target genes was only recently discovered. MicroRNAs (miRNAs) represent a novel class of regulatory gene products. The synthesis of their precursors closely resembles that of mRNAs, with many miRNAencoding regions embedded in regular protein-coding genes. miRNAs are then processed from such precursors through specific nucleases. Rather than encoding proteins, they act as regulators of mRNA stability and/or protein synthesis through specific hybridization of their seed sequence with mRNA target sequences, allowing each miRNA species to regulate a characteristic set of mRNAs. ${ }^{8,9}$ Multiple links between miRNA activity and cancer have been established. ${ }^{10,11}$ Strikingly, p53 was found to induce the expression of some miRNAs. ${ }^{12-14}$ Most notably, the miR-34 family of miRNAs contributes to apoptosis on induction by $\mathrm{p} 53 .{ }^{15-20}$

These findings raise the question whether E2F1 may also induce miRNAs that contribute to apoptosis. Indeed, E2F1responsive miRNAs were previously ${ }^{21,22}$ identified and at least partially characterized according to their role in cancer, but mostly with antiapoptotic functions ${ }^{23-25}$ or with no reported influence on apoptosis. To identify a more complete set of E2F1-inducible miRNAs, we performed array hybridization and found miR-449a and miR-449b (collectively termed miR449 from here on) to be strongly E2F1-responsive, in agreement with a recent report. ${ }^{22}$ Strikingly, miR-449 shares

\footnotetext{
${ }^{1}$ Department of Molecular Oncology, Göttingen Center of Molecular Biosciences -GZMB, Ernst Caspari Haus, University of Göttingen, Justus von Liebig Weg 11, 37077 Göttingen, Germany and ${ }^{2}$ Research Group Developmental Biology, Max Planck Institute for Biophysical Chemistry, Am Fassberg, 37077 Göttingen, Germany *Corresponding author: M Dobbelstein, Department of Molecular Oncology, GZMB, Ernst Caspari Haus, University of Göttingen, Justus von Liebig Weg 11, Göttingen D-37075, Germany. Tel: + 004955139 13840; Fax: + 004955139 13713; E-mail: mdobbel@ uni-goettingen.de Keywords: E2F1; p53; microRNA; miR-449; miR-34; DNA damage Abbreviations: Rb, retinoblastoma; cdks, cyclin-dependent kinases; miRNAs, microRNAs Received 29.10.09; accepted 05.11.09; Edited by G Melino; published online 04.12.09
} 


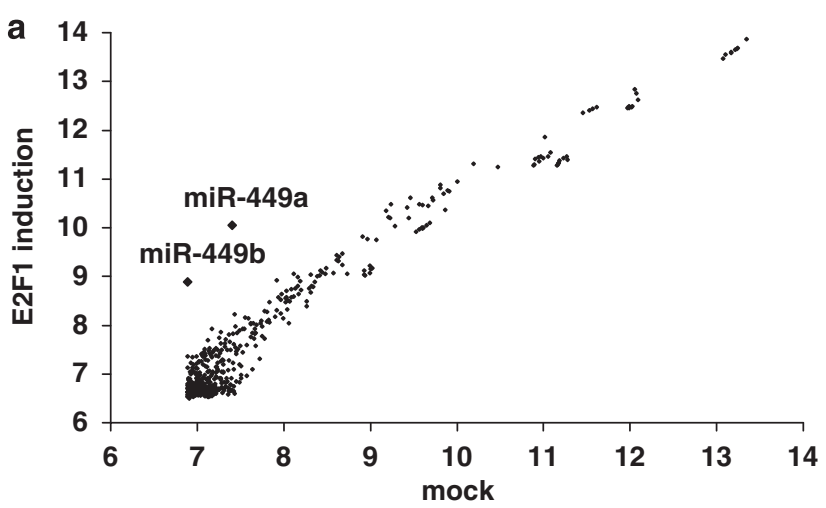

b

miR-449a

UGgCAgUGUAUUGUUAGCUGGU

miR-449b AGGCAGUGUAUUGUUAGCUGGC

miR-34a

miR-34b*

UGGCAGUGUCUUAGCUGGUUGU

miR-34c

UAGGCAGUGUCAUUAGCUGAUUG

AGGCAGUGUAGUUAGCUGAUUGC

Figure 1 E2F1-induced microRNAs identified by microRNA hybridization. Saos-2 cells with an integrated tet-inducible expression cassette to synthesize $\mathrm{E} 2 \mathrm{~F} 1^{26}$ were treated with doxycycline or were mock treated. At $24 \mathrm{~h}$ after infection, the cells were harvested. (a) MicroRNAs from each sample were fluorescencelabelled and hybridized to a microarray. The fluorescence intensity corresponding to each microRNA with or without E2F1 overexpression is shown plotted against each other. (b) Sequence of miR-449, a and b, aligned with miR-34, a, b and c. The shared seed sequence is shown in bold

seed sequences and target genes with the miR-34 family. miR-449 potently induces apoptosis and also upregulates p53 activity. However, it also attenuates E2F1 and the same was found for miR-34a. Thus, miR-449 and miR-34 precisely reflect the interdependence of E2F1 and p53 activity. Although E2F1-induced miR-449 and p53-induced miR-34 promote $\mathrm{p} 53$ activity and apoptosis, they negatively regulate E2F1. Hence, the influence of E2F1 and p53 on each other and on cell fate decisions is sustained by the induction of two miRNA species from the same family.

\section{Results}

E2F1 regulates miRNA expression. To identify E2F1responsive miRNAs, we employed Saos2 cells with tetracycline/doxycycline-inducible E2F1 ${ }^{26}$ (tet-on E2F1), controlled for E2F1 and E2F1 target gene expression (Supplementary Figure $\mathrm{S} 1 \mathrm{~A}$ ) and isolated a fraction enriched in small RNAs before and after doxycycline treatment. Subsequent fluorescence labeling and array hybridization identified a number of E2F1-responsive miRNAs, some of which have been described as such earlier (Figure 1a, Table 1 and Supplementary Table 1), for example, members of the miR-17-92 or $106 \mathrm{~b}-25$ clusters. $^{21,23,27}$ The strongest response was observed with a pair of closely related miRNAs, miR-449a and miR-449b, recently reported as E2F1-inducible miRNAs, $^{22}$ and
Table 1 Relative increase in miRNA levels on E2F1 expression, as revealed by microarray analysis

miRNA

Fold induction by E2F1

\begin{tabular}{ll}
\hline miR-449a & 6.3 \\
miR-449b & 4.1 \\
miR-492 & 2.4 \\
let-7b & 2.2 \\
let-7c & 2.2 \\
miR-663 & 2.2 \\
miR-19b & 2.1 \\
miR-30b & 2.0 \\
miR-16 & 2.0 \\
miR-762 & 2.0 \\
miR-17 & 2.0 \\
miR-106a & 2.0 \\
miR-23a & 2.9 \\
miR-20a & 1.9 \\
miR-106b & 1.9
\end{tabular}

MicroRNA levels were compared by microarray hybridization before and after induction of E2F1, as outlined in the legend to Figure 1a. The microRNAs most strongly upregulated by E2F1 are shown in the Table. A complete data set is provided in Supplementary Table S1.

collectively referred to as miR-449 from here on. These miRNAs show strong similarities to miRNAs miR-34a-c, especially within their $5^{\prime}$ portion that is generally believed to act as a 'seed sequence' that determines the specificity of target $\mathrm{mRNAs}^{9}$ (Figure 1b). As miR-449a levels by far exceed those of miR-449b in all systems analyzed so far (Figure 1a and see Landgraf et $a{ }^{28}{ }^{28}$ ), we focussed our further analysis on miR-449a.

E2F1 augments miR-449a levels. The enhanced expression of miR-449a in response to E2F1 was first confirmed by reverse transcription PCR (RT-PCR) in doxycycline-treated Saos2 cells with tet-on E2F1, whereas doxycycline did not enhance miR-449-levels in the parental Saos2 cells (Figure 2a). Moreover, a transiently transfected expression plasmid for E2F1 induced miR-449a in H1299 and U2OS cells, ensuring E2F1 responsiveness as a more general property of miR-449a. Finally, DNA damage by Camptothecin, a known trigger of E2F1 activity, ${ }^{7}$ enhanced miR-449a expression regardless of the p53 status. In contrast, E2F1 induced miR-34a far less efficiently than miR-449a (Supplementary Figure S1B). Conversely, p53 activation by the pharmacological Mdm2 antagonist Nutlin-3a, ${ }^{29}$ although capable of inducing miR-34a, ${ }^{12}$ failed to increase the levels of miR-449a (Supplementary Figure S1C).

miR-449 expression is coupled to its host gene CDC2OB and reduced in tumor cells. Next, we sought to determine the pattern of miR-449 expression in various tissues and tumor cells. The genomic region encoding both miR-449a and miR-449b is embedded into an intronic sequence of the mRNA-encoding gene $C D C 20 B$, a paralog of CDC2O (Figure 3a). RT-PCR analysis revealed that $C D C 20 B$ was induced by E2F1 as well (Supplementary Figure S1A). High levels of miR-449 were found in lung, trachea and testis, and still considerable amounts in cervix and oesophagus; the expression pattern of $C D C 20 B$ largely reflected the levels of miR-449 (Figure 3b). Mir-449 thus seems to be regulated through the activation of its host gene. High levels of miR- 

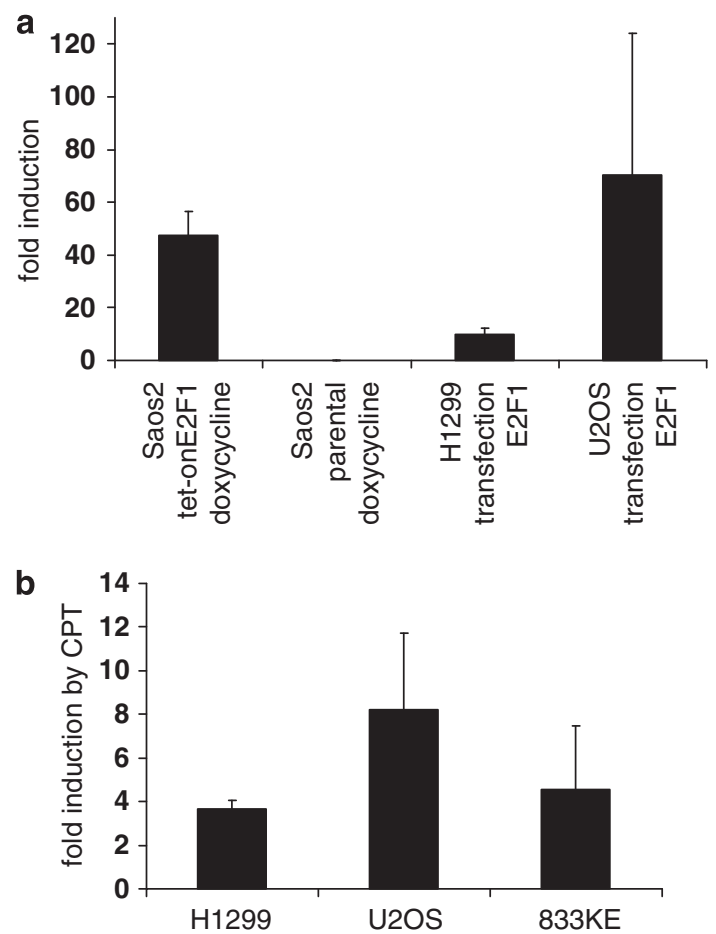

Figure 2 E2F1 overexpression and DNA damage induce miR-449. (a) RNA was extracted from Saos2-tet-E2F1 cells with or without earlier doxycycline treatment, or from $\mathrm{H} 1299$ or U2OS cells transfected with empty vector or an E2F1 expression plasmid. The levels of miR-449a were determined by RT-PCR analysis and normalized relative to a constitutively expressed small RNA, RNU6b. The ratio of miR-449a levels with versus without E2F1 overexpression was calculated from three independent experiments along with the standard error in each case. (b) MiR449 a induction by DNA damage. Cells of the indicated lines ( $\mathrm{H} 1299$, lung adenocarcinoma, $\mathrm{p} 53^{-1-}$, U2OS, osteosarcoma, p53 wild type; 833KE, testicular cancer and p53 mutant) were treated with camptothecin (CPT) followed by RT-PCR analysis of miR-449a levels as in a

449a in testis, lung and trachea were again found in murine tissues (Figure 3c). In contrast to testicular tissue, all tumorderived cell lines under study, including testicular cancer cells (GH and 833KE) and a lung adenocarcinoma cell line (H1299), contained far lower amounts of miR-449 (Figure 3d), at least compatible with the idea that miR-449 may display tumor-suppressive activities. Along this line, we found that trichostatin A, a pharmacological inhibitor of histone deacetylases, strongly increased miR-449 levels in cell lines from testicular cancer, although less efficiently in other cell lines (Supplementary Figure S2). This argues that epigenetic silencing may lead to miR-449 downregulation in the corresponding tumor cells.

miR-449 induces apoptosis. E2F1 is a potent inducer of apoptosis, especially in the context of DNA damage. The E2F1-responsive miR-449 closely resembles miR-34, a p53responsive and proapoptotic miRNA family. Hence, we tested whether miR-449 may act in parallel to miR-34 in growth suppression and apoptosis, as suggested by its activity in prostate cancer cells. ${ }^{30}$ First, we transfected plasmids encoding each miRNA into HCT116 cells with or without $\mathrm{p53}$, followed by antibiotic selection of stable transfectants. Both miR-34a and miR-449a/b suppressed clonogenic survival in this system, in a partially p53independent fashion (Figure $4 a$ and $b$ ). To further elucidate the mechanistic reason behind this suppressive effect, we analyzed the DNA content of transiently miRNA-transfected cells by flow cytometry and found that miR-34a and miR449a each led to cell accumulation in a sub-G1 shoulder (Figure 4c and Supplementary Figure S3, A and B), arguing that both miRNAs induce apoptosis. Under the same conditions, cleaved poly-ADP-ribose polymerase (PARP) and cleaved caspase 3 were detected by immunoblot analysis (Figure 4d), further supporting this notion. Although apoptosis induction seemed somewhat weaker in cells lacking p53, it was still observed in HCT116 p53 ${ }^{-/-}$cells and also in H1299 cells that do not contain p53 (Supplementary Figure S3C).

miR-449a and miR-34a are each capable of reducing CDK6 and Sirt1 levels, while inducing p53 acetylation. To identify possible mechanisms for miR-449-induced cell death, we analyzed putative target genes for miR-449a, taking into account the shared seed sequence with miR-34a. Although a complex pattern of gene regulation has previously been reported for miR-34a, ${ }^{15-20}$ we assumed that a few profoundly regulated target mRNAs may still serve as key targets to mediate the biological functions of miR-34a and possibly also miR-449. Accordingly, both miR-449a and miR-34a were found capable of downregulating the histone deacetylase Sirt1 in HCT116 cells with or without p53 (Figure $5 a$ and b) and in H1299 cells (Supplementary Figure S3C). Negative regulation was also observed for Sirt1 mRNA (Supplementary Figure S4); this suggests that regulation might occur through mRNA destabilization, but it does not exclude translational inhibition as an additional mechanism. The suppression of Sirt 1 levels by miR-449a is in agreement with software-based predictions (Targetscan, Cambridge, MA, USA and Miranda, New York, NY, USA) and previous reports on miR-34a. ${ }^{31}$ As Sirt1 is known to affect the acetylation of p53 at residue Lys $382^{31,32}$ we further analyzed the levels of acetylated p53 and also of the p53 target gene product p21. Both p21 and acetyl-p53 were upregulated, despite unchanged levels of global p53 and phospho-Ser15-p53 (Figure 5a). In addition to this, however, phosphorylated Histone $2 \mathrm{AX}$ was detected at increased levels under such circumstances, arguing that miR-449a and miR-34a are perhaps capable of increasing the susceptibility of cells to DNA damage. Phospho-H2AX accumulation in response to miR-449a was also observed in U2OS cells (data not shown) and only weakly in H1299 cells (Supplementary Figure S3C). Although not fully explained at present, this would be in line with the known ability of E2F1 to induce a DNA damage response and with similar responses to oncogenic stress during early cancerogenesis. ${ }^{33}$

In addition to these regulatory events, we also tested the effects of miR-449a and miR-34a on CDK6, another predicted target gene product. ${ }^{22,34}$ CDK6 was strongly downregulated under such circumstances (Figure $5 \mathrm{a}$ and $\mathrm{b}$ and Supplementary Figure S4). This argues that miR-449a provides a negative feedback loop that attenuates E2F1 activity even in situations in which cell death can be avoided. A reduction of 

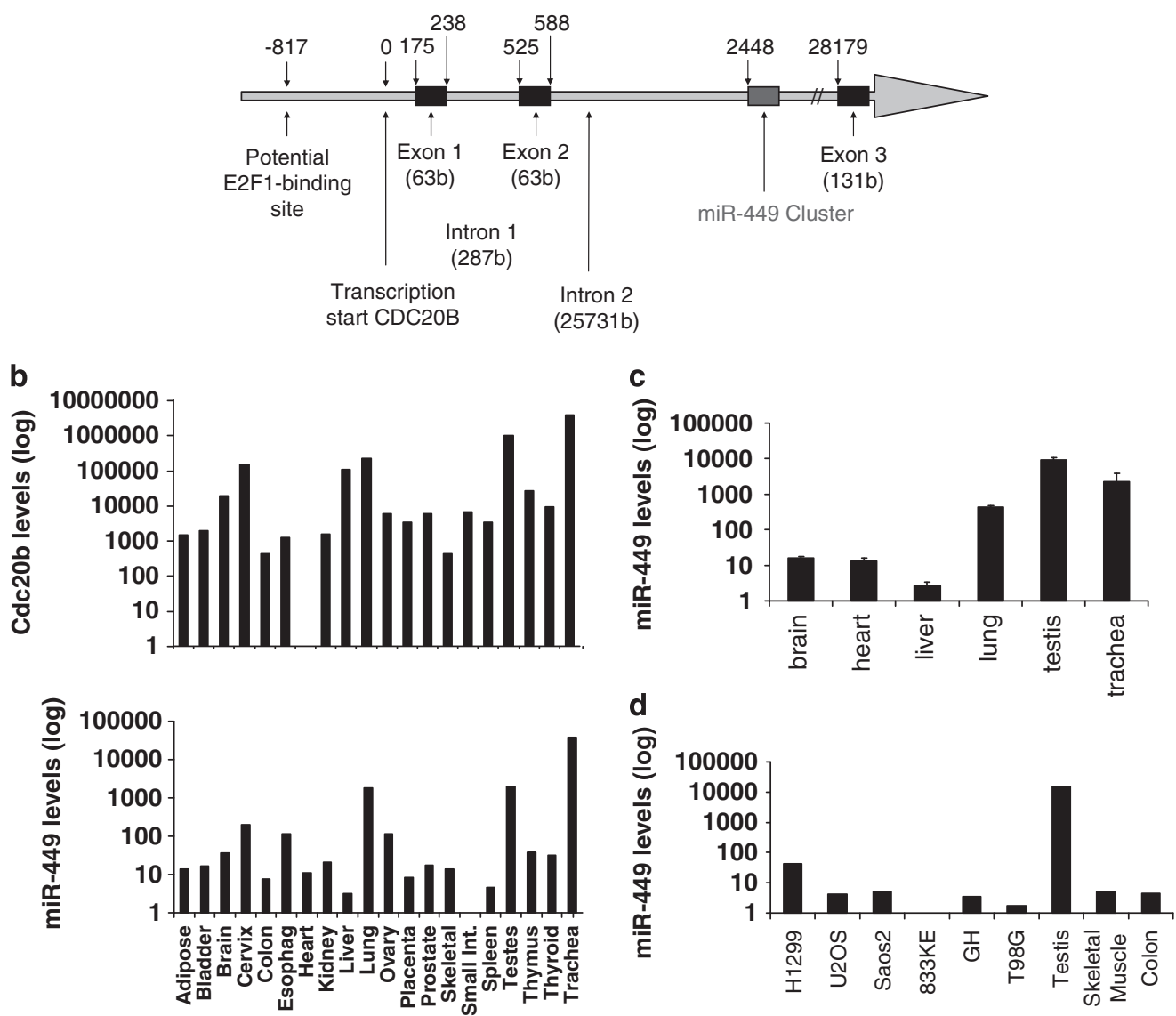

Figure $3 \mathrm{miR}-449 \mathrm{a}$ levels are reduced in tumor cells, and their regulation pattern resembles the expression of CDC20B. (a) Position of the miR-449 cluster within the CDC20B gene. (b) Parallel expression of CDC20B and miR-449a in a variety of human tissues (RNA panel, Ambion), as quantified by RT-PCR. (c) MiR-499a expression levels in murine tissues. (d) MiR-499a levels in human cancer-derived cell lines, as compared with human tissues

CDK6 is in line with the observed decrease of cells in S-phase (Figure 4b), presumably through hypophosphorylation and consecutive activation of $\mathrm{Rb}$ family pocket proteins. miR-449 may thus prevent the accumulation of cells with excessive E2F1 activity in two ways: providing a direct negative feedback on E2F1 activity and eliminating cells with uncontrollable E2F1.

\section{Discussion}

The results reported here strongly suggest a dual role for miR449. It provides a negative feedback to E2F1 activity, in part through targeting CDK6. However, the most obvious activity of miR-449 that we observed consists in the induction of apoptosis, in analogy to miR-34.

These findings are summarized in the model presented in Figure 6. Both p53 and E2F1 are activated in response to DNA damage and they subsequently induce proapoptotic genes. However, the mutual regulation of E2F1 and p53 is asymmetric. E2F1 induces the expression of p14arf and TAp73, each leading to enhanced induction of p53 target genes. On the other hand, p53 transactivates the gene encoding p21, a cdk inhibitor that negatively regulates E2F1
(Figure 6a). The activities of miRNAs reported here are in analogy to this model. First, miR-34 and miR-449 are each capable of promoting apoptosis, presumably by antagonizing an overlapping set of prosurvival genes. In addition to this, both miRNAs activate p53 while attenuating E2F1 (Figure 6b). This strongly argues that miRNAs of the 34/449 group fortify both the proapoptotic activities of p53 and E2F1, and also the asymmetric mutual regulation of these two factors.

As miR-34a and miR-449a can each reduce the expression levels of CDK6, it seems plausible how they limit cell cycle progression. In cells that contain wild-type p53, this effect seems enhanced through the upregulation of p21 (Figure 6a). However, it is less obvious how both miRNAs can induce apoptosis. Both miRNAs upregulate p53 acetylation (Figure 5a) and activity (Figure $5 \mathrm{a}$ and see Yamakuchi et $\left.a .^{31}\right)$. But as both miRNAs promote cell death in $\mathrm{p} 53^{-1-}$ cells, they must also trigger proapoptotic mechanisms independently of $\mathrm{p} 53$. We speculate that the negative regulation of the deacetylase Sirt1 (Figure 5) at least contributes to cell death, perhaps in concert with HDAC1 reduction; ${ }^{30}$ this may at least in part occur through the accumulation of DNA damage, as described earlier as a result of Sirt1 downregulation. ${ }^{35}$ It should be noted, however, that 
a

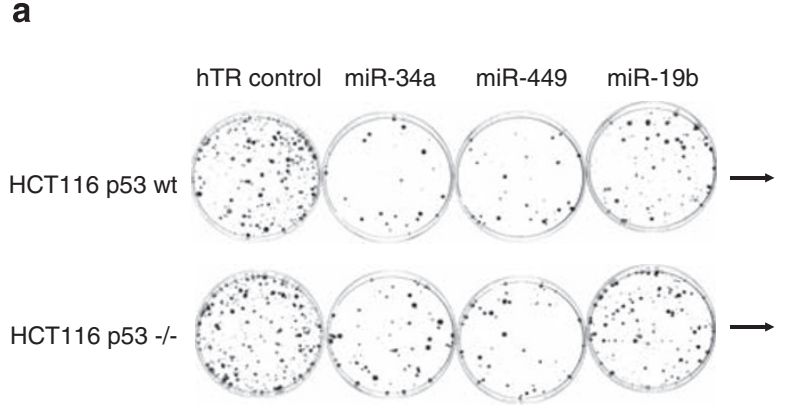

b
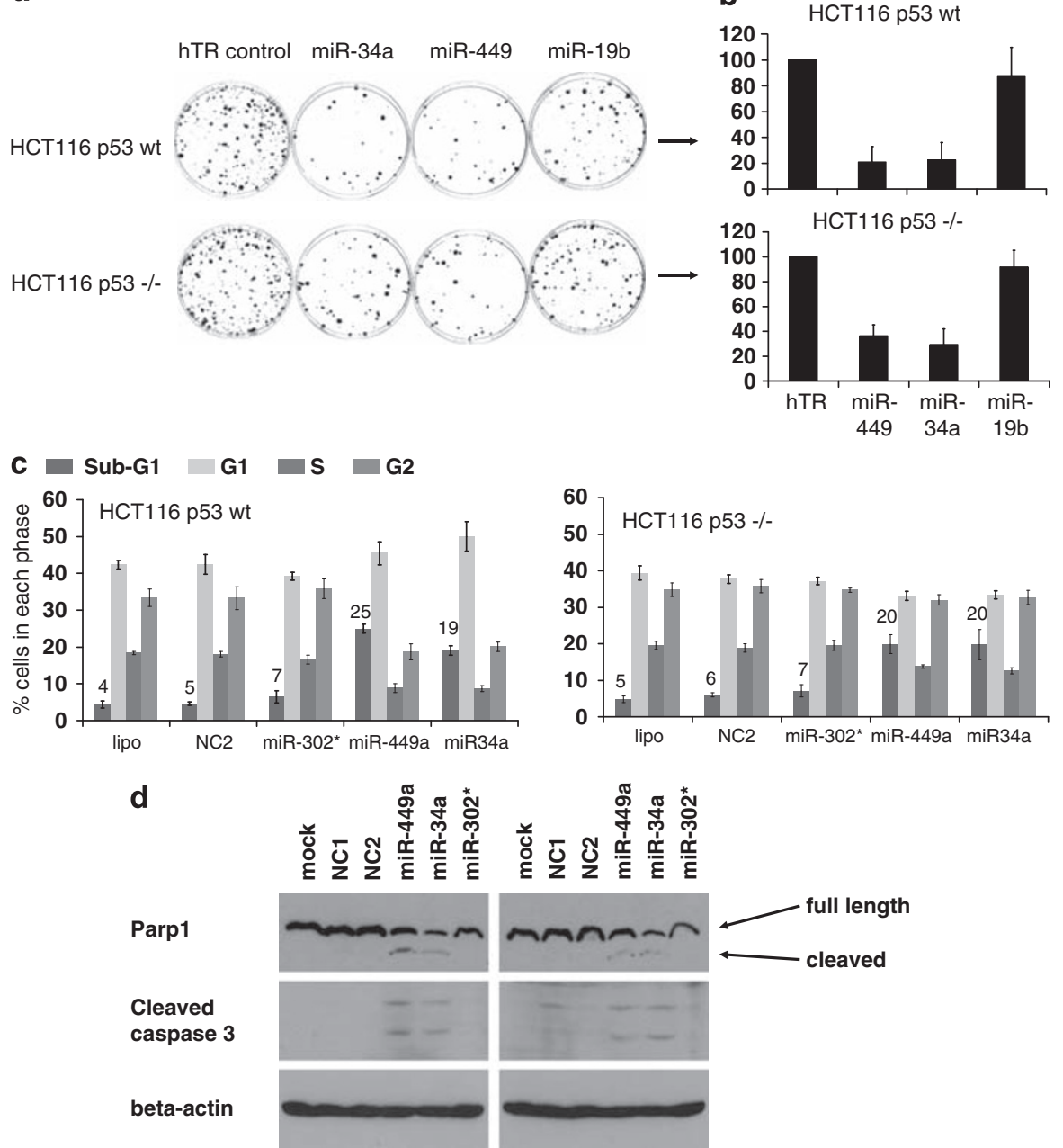

Figure 4 miR-449 induces apoptosis. (a) Reduced clonogenic survival on continuous expression of miR-449 and miR-34a. HCT116 cells with or without p53 were transfected with expression plasmids for miR-449a/b, miR-34a, or with control plasmids, followed by Blasticidin selection of stably transfected cells, as described previously. ${ }^{12}$ Two weeks after transfection, colonies were fixed and stained with crystal violet. (b) Quantification of the assays shown in a, performed by digitally determining the area covered by crystal violet-stained cells. The results of five independent experiments are summarized along with the standard error in each case. (c) Induction of a sub-G1 shoulder and decreased S-Phase fraction by miR-449a and miR-34a. HCT116 cells with or without p53 were transiently transfected with synthetic miR-449a, miR-34a or control miRNAs. At $48 \mathrm{~h}$ post transfection, the cells were fixed, stained with propidium iodide and subjected to flow cytometry. The percentage of (presumably apoptotic) cells in the sub-G1 fraction was calculated in each case based on the distribution of staining intensities (exemplified in Supplementary Figure S4). Results from three independent experiments are shown along with the standard error. (d) Induction of caspase activity by miR-449a and miR-34a. HCT116 cells were treated as in $\mathbf{b}$, followed by immunoblot analysis of poly-ADP ribose polymerase (PARP); the lower band is characteristic of a caspase-derived cleavage fragment. Cleaved caspase 3 was stained by another antibody

the knockdown of Sirt1 alone did not phenocopy the proapoptotic effects of miR-34 or miR-449 under the conditions used in our study, although it did suppress clonogenic survival (our unpublished results). Nonetheless, this does not exclude a contribution of Sirt1 downregulation to the observed cell death when combined with broader gene regulation. Extensive studies on miR-34 target genes have revealed a multitude of potential survival genes as being miR-34 regulated. ${ }^{15,17}$ We assume that miR-449 has a similarly broad spectrum of target mRNAs, largely excluding a monocausal model to explain its proapoptotic effects.

Both miR-449a and miR-449b are encoded by an intron of the $C D C 20 B$ gene. Although little is known about the function of this gene, its paralog $C D C 20$ is an essential component of the cellular machinery that enables the anaphase-promoting complex to destruct its targets in a timely manner, allowing progression through mitosis. ${ }^{36}$ It is to be noted that several mitosis-promoting genes, for example, Polo-like kinase and others, have previously been identified as E2F1 targets, implying E2F1 in mitotic progression. ${ }^{37}$ It is tempting to speculate that E2F1 may further promote mitosis through CDC20B, whereas uncontrolled E2F1 activity is avoided by building a negative feedback into the same gene.

Tumor cells generally appear to contain low levels of miR449. The cells may well be selected for miR-449 silencing, as they may otherwise undergo cell cycle arrest or apoptosis. The fact that E2F1 activity is frequently deregulated, for example, through deleting the $\mathrm{pRb}$ gene, may further increase the need for miR-449 silencing to allow tumor cell survival. HDAC inhibition seems to upregulate miR-449, and this may 


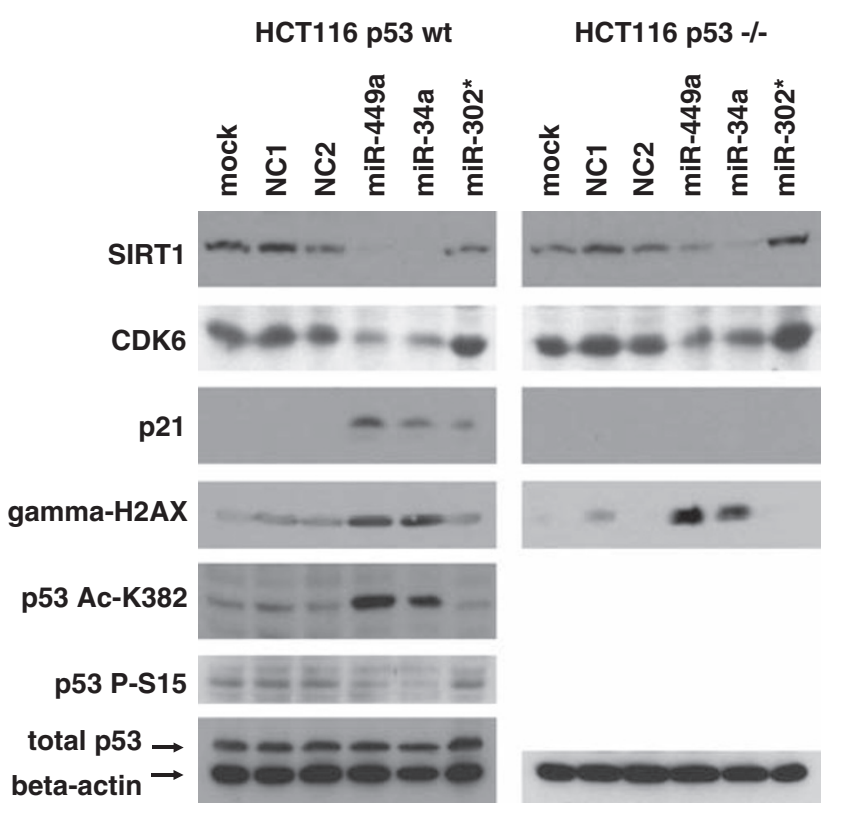

Figure 5 miR-449 reduces CDK6 and Sirt1 levels and augments the acetylation of p53. HCT116 p53 wild type (wt) or HCT116 p53 $3^{-1-}$ cells were transfected with the indicated microRNAs, followed by immunoblot analysis to detect the indicated gene products and modifications with specific antibodies. P53 staining was only performed in HCT116 p53 wt cells (but lack of p53 was confirmed in HCT116 $\mathrm{p} 53^{-1-}$ cells in additional experiments, data not shown)

be further enhanced by the reversal of DNA methylation. ${ }^{22}$ This may represent one of the (numerous) mechanisms that explain cell death on HDAC inhibitor treatment. Tumor cells are generally prone to a constitutive DNA damage response ${ }^{33}$ and apoptosis by oncogenic stress. Thus, they can be expected to undergo apoptosis in response to miR-449 or miR-34 at an enhanced rate, rather than merely arresting proliferation. Residual expression levels of miR-449 and miR-34a, and their inducibility by E2F1 and p53, may thus represent a determinant for cancer cell death in response to irradiation or chemotherapy.

The E2F family is essential for cell proliferation. However, its uncontrolled activity is one of the most widely acknowledged reasons for malignant growth. MiR-449 contributes to the due control in two ways, by antagonizing E2F1 and also by eliminating the cell when necessary. As both mechanisms can occur independently of p53, miR-449 may represent an essential barrier to cancer progression in cells that have already lost p53 function through mutations.

\section{Materials and Methods}

Cell culture and drug treatment. HCT116 cells with and without p53 (obtained from B Vogelstein ${ }^{38}$ ) were grown in McCoy's 5A, H1299 (from A Levine), U2OS (LGC/ATCC, Wesel, Germany), GH and 833KE (from R Löwer) and Saos-2 cells without (ATCC) or with tet-inducible E2F1 (from K Vousden ${ }^{26}$ ) were cultivated in Dulbecco's modified Eagle's medium, each supplemented with $10 \%$ fetal bovine serum. Camptothecin was used at $2.9 \mu \mathrm{M}$. Plasmid transfections were carried out using Lipofectamine 2000.

miRNA microarray analysis to identify E2F1-responsive miRNAs. Saos2 cells with an integrated, inducible ('tet-on') E2F1 expression construct $^{26}$ were treated or not with doxycycline at a concentration of $2 \mu \mathrm{g} / \mathrm{ml}$ for

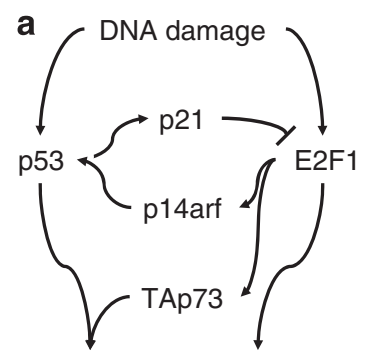

Pro-apoptotic genes

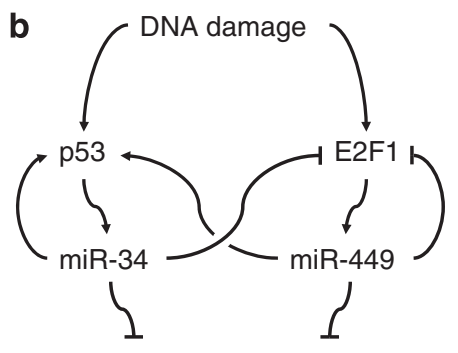

Pro-survival genes
Figure 6 Model for mutual regulation of p53 and E2F1. (a) Mutual regulation through protein-coding target genes. DNA damage activates p53 and E2F1, and both induce an overlapping set of proapoptotic target genes. Although E2F1 further enhances p53 activity through p14arf and TAp73, p53 inhibits E2F1 through the CDK inhibitor p21. (b) This asymmetric mutual regulation is recapitulated and presumably fortified by microRNAs. On DNA damage, p53 induces miR-34 and E2F1 increases the levels of miR449 family members. Both microRNAs induce apoptosis by antagonizing the expression of prosurvival genes. In addition to this, both microRNAs support p53 activity (for example, through enhanced acetylation), but negatively regulate E2F1 (for example, by downregulating $\mathrm{CDK} 6$ ). Arrowheads symbolize activation and bars inhibition.

$24 \mathrm{~h}$. RNA samples enriched for small RNA molecules were isolated by using the miRVana RNA Isolation kit (Ambion/Applied Biosystems, Darmstadt, Germany). Array hybridization was carried out using the Exiqon system of arrayed locked nucleic acids (Vedbaek, Denmark), as recommended by the manufacturer.

RNA extraction and quantitative RT-PCR (qRT-PCR) analysis. For mRNA and miRNA-analyses, total RNA was isolated using the mirVana RNA Isolation kit (Ambion). For mRNA analysis alone, total RNA was isolated by using TRIzol (Invitrogen, Paisley, UK). To detect mRNA expression, the isolated RNA was reverse transcribed using the iscript CDNA Synthesis Kit (Bio-Rad, Munich, Germany). IQ SYBR Green Supermix (Bio-Rad) was used for real-time PCR applications. The qRT-PCR primer set for CDC20B was $5^{\prime}$-GGAGATGCTGTGGGA AAGTA-3' and $5^{\prime}$-CGCAAAGTTGCTCTTAAAGTC-3'; for CDK6, 5'-AGACCCAA GAAGCAGTGTGG-3' and 5'-AAGGAGCAAGAGCATTCAGC-3'; for E2F1, 5'-CG GTGTCGTCGACCTGAACT- $3^{\prime}$ and $5^{\prime}$-AGGACGTTGGTGATGTCATAGATG-3' ${ }^{\prime}$, for SIRT1, 5' -GAGATAACCTTCTGTTCGGTG- $3^{\prime}$ and $5^{\prime}$-CGGCAATAAATCTTTAAGA AT-3'; and for TAp73 5'-CACCACGTTTGAGCACCTCTG-3' and 5'-GATGTAGTCA TGCCCTCCAGG- $3^{\prime}$.

qRT-PCR data were normalized to mtRNR2a (primer set: $5^{\prime}$-CATAAGCC TGCGTCAGATCA-3' and 5'-CCTGTGTTGGGTTGGACA-3') or 36B4 (primer set: $5^{\prime}$-GATTGGCTACCCAACTGTTG-3' and $5^{\prime}$-CAGGGGCAGCAGCCACAAA- $3^{\prime}$ ), as shown in Supplementary Figure S4. Stem-loop qRT-PCR for mature miRNAs was carried out using the TaqMan miRNA assays (Applied Biosystems), as described. ${ }^{12,39}$ miRNA expression levels were normalized to RNU6B or to U6 when analyzing murine tissue. The standard curve method was used for the relative quantification of gene expression.

Transient transfection of cells with pre-miRNAs. Cells were reverse transfected with synthetic pre-miRNAs (Ambion/Applied Biosystems). miRNA was diluted in $250 \mu \mathrm{l}$ medium without serum at $10 \mathrm{nM}$ concentration. Lipofectamine 2000 ( $5 \mu \mathrm{l}$; Invitrogen) was incubated with $250 \mu \mathrm{l}$ medium for $5 \mathrm{~min}$. The combination of both mixtures was incubated for $20 \mathrm{~min}$ at room temperature and added to a suspension of freshly trypsinized cell followed by seeding in six-well dishes.

Clonogenic assays. HCT116 cells were transfected with MiR vectors, a generous gift from R. Agami, ${ }^{40}$ in six-well plates. Cultures were maintained for 2 weeks with Blasticidin $(10 \mu \mathrm{g} / \mathrm{ml})$, followed by fixation and staining, as described previously. ${ }^{12}$

Flow cytometry. The attached cells combined with floating cells were harvested and fixed in $70 \%$ ethanol at $4{ }^{\circ} \mathrm{C}$. The cells were then resuspended and incubated for $30 \mathrm{~min}$ in PBS containing $1 \mathrm{mg} / \mathrm{ml}$ RNase $A$ and stained with $15 \mu \mathrm{g} / \mathrm{ml}$ propidium iodide (end concentration). Samples were analyzed by the 
Guava/Millipore (Billerica, MA, USA) Easycyte Plus System using the Guava ExpressPro or ModFit Software (Champaign, IL, USA).

Immunoblot analysis. After SDS-polyacrylamide gel electrophoresis and wet transfer to nitrocellulose, the membranes were blocked and incubated with antibodies in PBS containing $5 \%$ non-fat milk powder or $5 \%$ bovine albumin fraction V. Primary antibodies used for detection were anti-acetyl-Lys382 p53, anti-CDK6 (DCS83), anti-cleaved caspase-3 (Asp175) (5A1) and anti-phospho-Ser15 p53 (16G8) (Cell Signaling Technology, Danvers, MA, USA); anti-p21 (EA10) and antiParp-1 (Ab-2) (all from Calbiochem/Merck, Darmstadt, Germany); anti-p53 (D01) and anti-SIRT1 (B-7) (each from Santa Cruz Biotechnology, Santa Cruz, CA, USA); anti-phospho-histone H2A.X (Ser139) (JBW301, Upstate, Billerica, MA, USA); and anti- $\beta$-actin (Abcam, Cambridge, MA, USA). Detection was performed by HPRconjugated antibodies (Jackson Immuno Research Laboratories, West Grove, PA, USA) and chemiluminescence (Millipore) on Super RX films (Fujifilm, Europe/ Fujifilm Deutschland, Düsseldorf, Germany).

Murine tissue analysis. Male $\mathrm{B} 6 \mathrm{~N}$ mice $(n=4)$ were used for tissue analysis. At the age of 10 weeks, the mice were killed and organs were isolated. The tissue was homogenized in $1 \mathrm{ml}$ of TRIzol (Invitrogen) and frozen in liquid nitrogen. After thawing, the lysate was chloroform-extracted and ethanol-precipitated. The pellet was dissolved in pre-heated miRVana (Ambion) elution buffer.

\section{Conflict of interest}

The authors declare no conflict of interest.

Acknowledgements. We thank $\mathrm{K}$ Vousden for Saos2 cells with tet-inducible E2F1, R Agami for plasmids, R Löwer for testicular cancer cells, the transcriptome analysis unit at our University for array hybridization, F Köpper and X Zhang for help with RT-PCRs, C Hippel and A Dickmanns for excellent technical assistance and R Ficner, T Pieler, M Kessel and S Johnsen for helpful discussion. The work was supported by the Wilhelm Sander Stiftung, the German Cancer Aid/Dr. Mildred Scheel Stiftung, the EU 6th Framework Program (Integrated Project Active p53), and the German Research Foundation (DFG).

1. Bell LA, Ryan KM. Life and death decisions by E2F-1. Cell Death Differ 2004; 11: 137-142. 2. Polager S, Ginsberg D. E2F - at the crossroads of life and death. Trends Cell Biol2008; 18: 528-535.

3. Irwin M, Marin MC, Phillips AC, Seelan RS, Smith DI, Liu W et al. Role for the p53 homologue p73 in E2F-1-induced apoptosis. Nature 2000; 407: 645-648.

4. Stiewe T, Putzer BM. Role of the p53-homologue p73 in E2F1-induced apoptosis. Nat Genet 2000; 26: 464-469.

5. Bates S, Phillips AC, Clark PA, Stott F, Peters G, Ludwig RL et al. p14ARF links the tumour suppressors RB and p53. Nature 1998; 395: 124-125.

6. Hershko T, Ginsberg D. Up-regulation of $\mathrm{Bcl}-2$ homology 3 (BH3)-only proteins by E2F1 mediates apoptosis. J Biol Chem 2004; 279: 8627-8634.

7. Polager S, Ginsberg D. p53 and E2f: partners in life and death. Nat Rev Cancer 2009; 9 : 738-748.

8. Flynt AS, Lai EC. Biological principles of microRNA-mediated regulation: shared themes amid diversity. Nat Rev Genet 2008; 9: 831-842.

9. Filipowicz W, Bhattacharyya SN, Sonenberg N. Mechanisms of post-transcriptional regulation by microRNAs: are the answers in sight? Nat Rev Genet 2008; 9: 102-114.

10. Kumar MS, Lu J, Mercer KL, Golub TR, Jacks T. Impaired microRNA processing enhances cellular transformation and tumorigenesis. Nat Genet 2007; 39: 673-677.

11. Croce CM. Causes and consequences of microRNA dysregulation in cancer. Nat Rev Genet 2009; 10: 704-714

12. Braun CJ, Zhang X, Savelyeva I, Wolff S, Moll UM, Schepeler T et al. p53-Responsive microRNAs 192 and 215 are capable of inducing cell cycle arrest. Cancer Res 2008; 68: 10094-10104.

13. Georges SA, Biery MC, Kim SY, Schelter JM, Guo J, Chang AN et al. Coordinated regulation of cell cycle transcripts by p53-inducible microRNAs, miR-192 and miR-215. Cancer Res 2008; 68: 10105-10112.
14. Georges SA, Chau BN, Braun CJ, Zhang X, Dobbelstein M. Cell cycle arrest or apoptosis by p53: are microRNAs-192/215 and -34 making the decision? Cell Cycle 2009; 8: 680-681.

15. Chang TC, Wentzel EA, Kent OA, Ramachandran K, Mullendore M, Lee KH et al Transactivation of miR-34a by $\mathrm{p} 53$ broadly influences gene expression and promotes apoptosis. Mol Cell 2007; 26: 745-752.

16. Corney DC, Flesken-Nikitin A, Godwin AK, Wang W, Nikitin AY. MicroRNA-34b and MicroRNA-34c are targets of p53 and cooperate in control of cell proliferation and adhesion-independent growth. Cancer Res 2007; 67: 8433-8438.

17. He L, He X, Lim LP, de Stanchina E, Xuan Z, Liang Y et al. A microRNA component of the p53 tumour suppressor network. Nature 2007; 447: 1130-1134.

18. Hermeking H. The miR-34 family in cancer and apoptosis. Cell Death Differ 2009; 22 May 2009. e-pub ahead of print.

19. Tazawa $H$, Tsuchiya N, Izumiya M, Nakagama $H$. Tumor-suppressive miR-34a induces senescence-like growth arrest through modulation of the E2F pathway in human colon cancer cells. Proc Natl Acad Sci USA 2007; 104: 15472-15477.

20. Raver-Shapira N, Marciano E, Meiri E, Spector $\mathrm{Y}$, Rosenfeld N, Moskovits N et al. Transcriptional activation of miR-34a contributes to p53-mediated apoptosis. Mol Cell 2007; 26: 731-743.

21. Pickering MT, Stadler BM, Kowalik TF. miR-17 and miR-20a temper an E2F1-induced G1 checkpoint to regulate cell cycle progression. Oncogene 2009; 28: 140-145.

22. Yang X, Feng M, Jiang X, Wu Z, Li Z, Aau M et al. miR-449a and miR-449b are direct transcriptional targets of E2F1 and negatively regulate pRb-E2F1 activity through a feedback loop by targeting CDK6 and CDC25A. Genes Dev 2009; 23: 2388-2393.

23. Sylvestre Y, De Guire V, Querido E, Mukhopadhyay UK, Bourdeau V, Major F et al. An E2F/miR-20a autoregulatory feedback loop. J Biol Chem 2007; 282: 2135-2143.

24. Yan HL, Xue G, Mei Q, Wang YZ, Ding FX, Liu MF et al. Repression of the miR-17-92 cluster by $p 53$ has an important function in hypoxia-induced apoptosis. EMBO J 2009; 28: 2719-2732.

25. Petrocca F, Vecchione A, Croce CM. Emerging role of miR-106b-25/miR-17-92 clusters in the control of transforming growth factor beta signaling. Cancer Res 2008; 68 : 8191-8194.

26. Phillips AC, Ernst MK, Bates S, Rice NR, Vousden KH. E2F-1 potentiates cell death by blocking antiapoptotic signaling pathways. Mol Cell 1999; 4: 771-781.

27. Petrocca F, Visone R, Onelli MR, Shah MH, Nicoloso MS, de Martino I et al. E2F1regulated microRNAs impair TGFbeta-dependent cell-cycle arrest and apoptosis in gastric cancer. Cancer Cell 2008; 13: 272-286.

28. Landgraf $P$, Rusu M, Sheridan R, Sewer A, lovino N, Aravin A et al. A mammalian microRNA expression atlas based on small RNA library sequencing. Cell 2007; 129 . 1401-1414.

29. Vassilev LT, Vu BT, Graves B, Carvajal D, Podlaski F, Filipovic Z et al. In vivo activation of the p53 pathway by small-molecule antagonists of MDM2. Science 2004; 303: 844-848.

30. Noonan EJ, Place RF, Pookot D, Basak S, Whitson JM, Hirata H et al. miR-449a targets HDAC-1 and induces growth arrest in prostate cancer. Oncogene 2009; 28: 1714-1724.

31. Yamakuchi M, Ferlito M, Lowenstein CJ. miR-34a repression of SIRT1 regulates apoptosis. Proc Natl Acad Sci USA 2008; 105: 13421-13426.

32. Solomon JM, Pasupuleti R, Xu L, McDonagh T, Curtis R, DiStefano PS et al. Inhibition of SIRT1 catalytic activity increases p53 acetylation but does not alter cell survival following DNA damage. Mol Cell Biol 2006; 26: 28-38.

33. Bartkova J, Horejsi Z, Koed K, Kramer A, Tort F, Zieger K et al. DNA damage response as a candidate anti-cancer barrier in early human tumorigenesis. Nature 2005; 434: 864-870.

34. Sun F, Fu H, Liu Q, Tie Y, Zhu J, Xing R et al. Downregulation of CCND1 and CDK6 by miR-34a induces cell cycle arrest. FEBS Lett 2008; 582: 1564-1568.

35. Wang RH, Sengupta K, Li C, Kim HS, Cao L, Xiao C et al. Impaired DNA damage response, genome instability, and tumorigenesis in SIRT1 mutant mice. Cancer Cell 2008; 14: 312-323.

36. Yu H. Cdc20: a WD40 activator for a cell cycle degradation machine. Mol Cell 2007; 27: 3-16.

37. Ren B, Cam H, Takahashi Y, Volkert T, Terragni J, Young RA et al. E2F integrates cell cycle progression with DNA repair, replication, and G(2)/M checkpoints. Genes Dev 2002; 16: $245-256$

38. Bunz F, Dutriaux A, Lengauer C, Waldman T, Zhou S, Brown JP et al. Requirement for p53 and p21 to sustain G2 arrest after DNA damage. Science 1998; 282: 1497-1501.

39. Scheel AH, Beyer U, Agami R, Dobbelstein M. Immunofluorescence-based screening identifies germ cell associated microRNA 302 as an antagonist to p63 expression. Cell Cycle 2009; 8: 1426-1432.

40. Voorhoeve PM, le Sage C, Schrier M, Gillis AJ, Stoop H, Nagel R et al. A genetic screen implicates miRNA-372 and miRNA-373 as oncogenes in testicular germ cell tumors. Cell 2006; 124: 1169-1181. 